\title{
Vibration Analysis and Control of a Vibration Screed System for Asphalt Pavers
}

\author{
Vanliem Nguyen, Zhenpeng Wu and Beiping Zhang \\ School of Mechanical and Electrical Engineering, Hubei Polytechnic University, Huangshi 435003, China.
}

\author{
Zhang Jian Run \\ School of Mechanical Engineering, Southeast University, Nanjing 211189, China.
}

(Received 5 August 2019; accepted 3 January 2020)

To reduce shaking of a vibration screed system (VSS) and improve the paving performance of an asphalt paver (AP), the root-mean-square (RMS) acceleration responses at points on the front and rear screed floors are analyzed via an experimental method. A 3D nonlinear dynamic model of the VSS is also built to evaluate the influence of the dynamic parameters of the VSS on the compression efficiency, paving quality, and working stability of the AP based on the objective functions of the vertical, pitching, and rolling RMS values at the centre of gravity of the screed. The angular deviations, $\alpha$ and $\gamma$, of the tamper are then controlled to improve the paving performance. The research results show that the excitation frequency, $f_{t}$, and both angular deviations, $\alpha$ and $\gamma$, of the tamper strongly affect the paving performance. The compression efficiency is quickly enhanced, while both paving quality and working stability are significantly reduced with increasing the excitation frequency $f_{t}$ and reducing the angular deviations. $\alpha$ and $\gamma$. and vice versa. Additionally, the screed shaking and paving performance of the AP are remarkably improved by control of the angular deviations, $\alpha$ and $\gamma$, under different working conditions.

\section{INTRODUCTION}

The asphalt paver (AP) was one of the construction machines used to pave asphalt mixture on road surface construction rapidly and uniformly. ${ }^{1,2}$ Therefore, the vibration screed system of an asphalt paver (VSS-AP) was equipped with a vibrator screed and a couple of tamper mechanisms (compacting beams). ${ }^{3-5}$ The tamper was used to compress the asphalt mixture to become tighter and more uniform in density while the vibrator screed was used to improve smooth and the finish of the road surface construction. The paving performance of the AP was mainly assessed by three indexes of the compression efficiency, paving quality, and working stability. 1,6

The compression efficiency was affected not only by the operating parameters of the VSS but also by the asphalt materials and ground vibrations. ${ }^{1,7-12}$ The influence of density, temperature, and size of particles of the asphalt mixture on compression efficiency was studied. ${ }^{9,13-15}$ The studies showed that the temperature of the hot asphalt-mix greatly impacted on the asphalt density and compression efficiency. In order to achieve the desired density, the temperature of the hot asphalt-mix in the compression process was quickly analyzed by a fuzzy clustering technique. ${ }^{10}$ The errors of unequal compaction coverage, temperature, and compaction delay were then controlled based on the compaction monitoring system. ${ }^{16}$ The influence of the different temperature regions of the asphalt-mix on compression efficiency was analyzed by using a multi-sensor infrared temperature scanning bar system. ${ }^{17}$ Besides, the ground motions and vibrations could affect the performance of machines working on the ground, ${ }^{11,18}$ especially the elastoplastic ground soils. ${ }^{19}$ Additionally, with the operating parameters of the VSS, the influence of the compression forces, ${ }^{20}$ phase deflections, and excitation frequencies ${ }^{7,21}$ of the tamper on the smoothness of the pavement was also investigated. The results indicated that the vibration excitation of the tamper mainly affected the compression efficiency. However, in all the above research, only the vertical vibration with a quarter model of the VSS-AP was considered.

The paving quality and working stability of the AP were significantly affected by the excitation frequency of the tamper $\left(f_{t}\right)$ and of vibration screed $\left(f_{s}\right){ }^{2,6,22}$ Based on the $2 \mathrm{D}$ dynamic model of the $\mathrm{VSS},{ }^{3}$ the analysis results showed that the paving quality was better with the vibration excitation of $f_{t}$ from 10 to $20 \mathrm{~Hz}$ and of $f_{s}$ from 30 to $40 \mathrm{~Hz}$. The optimal paving performance was found at the excitation frequency $15 \mathrm{~Hz}$ of $f_{t}$ and $32 \mathrm{~Hz}$ of $f_{s}$ on a type of asphalt-mix materials. ${ }^{6}$ Three different types of asphalt-mix materials of SMA13, AC-20, and AC-25 were then expanded to fully analyze the influence of the excitation frequency of $f_{t}$ and $f_{s} \cdot{ }^{1,4,5}$ All researches showed that compression efficiency was significantly improved, but paving quality and working stability were still low. To solve this problem, the mass of tamper and eccentric distance of the eccentric shaft were optimized to reduce the vibration amplitude on all nodes of the screed which helped to improve the compression surface quality. ${ }^{23}$ Both the excitation frequencies of $f_{t}$ and $f_{s}$ were also optimized based on a genetic algorithm to decrease the pitching vibration $(\phi)$ of the screed. ${ }^{5}$ Besides, the angular deviation $(\gamma)$ between the front/rear tampers was also optimized via ADAM 


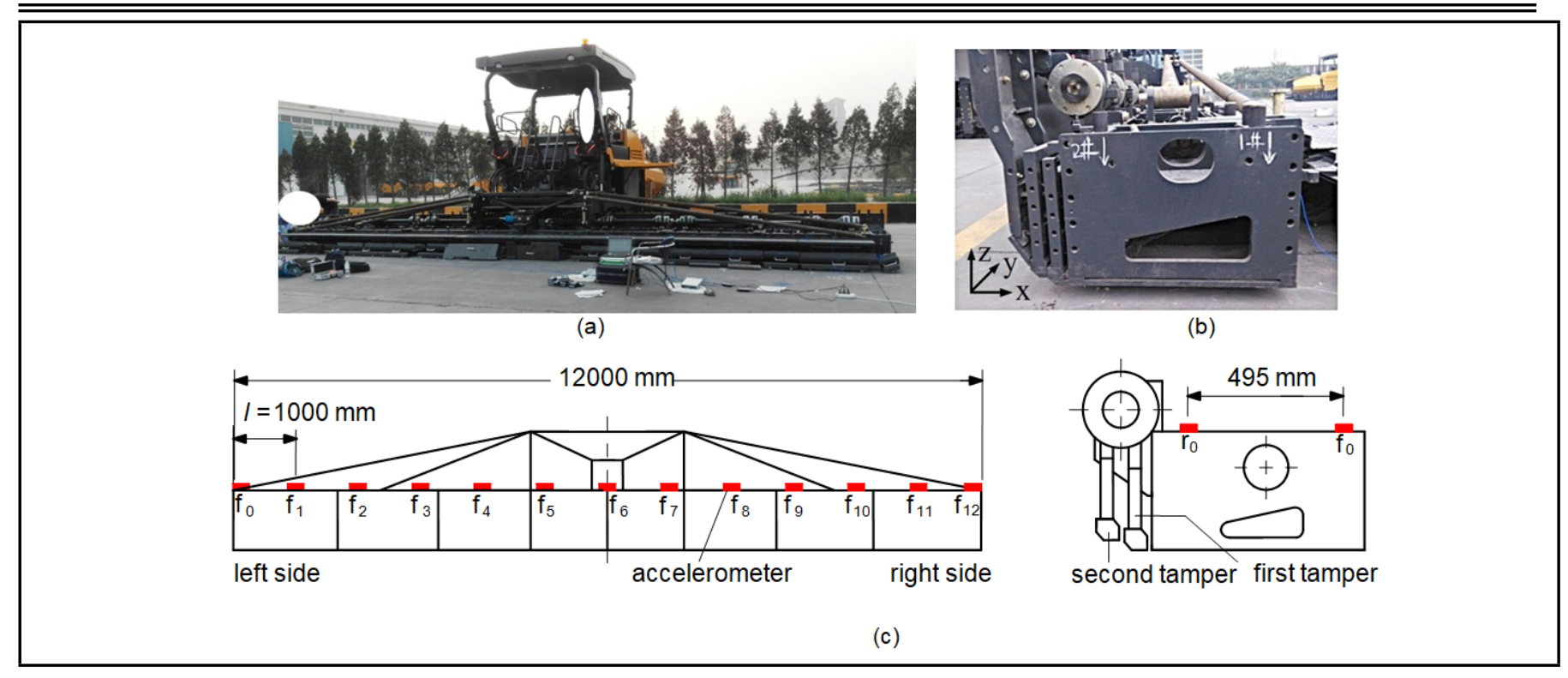

Figure 1. Experimental set-up for the Asphalt Paver (AP) (a), vibration screed system (b), and installation locations of accelerometers on the screed floor (c).

and ABAQUS software to improve the paving performance. ${ }^{6}$ The studies concluded that the angular deviations of the tamper significantly influenced the working stability apart from the excitation frequencies. Additionally, the actual width of the VSS was usually 9000 or $1200 \mathrm{~mm} .{ }^{3,6}$ Therefore, the rolling vibration $(\theta)$ of the screed, the angular deviations of excitation forces between the right and left sides $(\alpha)$ and between tampers $\left(\beta_{i}\right)$ of the tamper could greatly impact the paving performance of the AP. However, this problem has not been investigated yet.

To evaluate the influence of vibration on the VSS as well as the paving performance of the AP, the root-mean-square (RMS) of the acceleration response ${ }^{24}$ was used as the evaluation index. ${ }^{2,3,15}$ In this study, the experimental method is used to evaluate the paving performance of the AP. A 3D dynamic model is also built to analyze the influence of the dynamic parameters of the VSS on the paving efficiency based on the RMS acceleration responses at the centre of gravity of the screed. The dynamic parameters are then controlled to further improve the paving performance of the AP.

The point of this new study is the evaluation of the impact of excitation vibrations on the screed shaking which mainly causes the unevenness of the paving density and paving surface, whilst the dynamic parameters are controlled to enhance the paving performance of the AP.

\section{VIBRATION ANALYSIS OF THE VSS-AP}

\subsection{Experimental Model}

The VSS has been equipped with eight couples of tamper mechanisms, ${ }^{3-5}$ in which, four couples of the right tamper mechanisms and four couples of the left tamper mechanisms are symmetrically designed. The angular deviation $\beta$ between the excitation forces of tamper mechanisms is also symmetrically designed following the tamper mechanisms. With each couple of tampers, the angular deviation between the front and rear tampers is $\gamma$. Besides, the vibration excitation of the left and right tamper mechanisms is also deviated by a phase angle $\alpha$. Additionally, the mass of the eccentric configuration of the vibrator screed is installed at the centre of gravity of screed.

To analyze the effect of vibration excitations on the paving performance, an asphalt paver with a screed width of $12000 \mathrm{~mm}, 3 \mathrm{D}$ accelerometers ICP ${ }^{\circledR}$, and an analysis system of Belgium LMS have been used to measure the accelerations under screed floor. There are 24 sensors with their sampling frequency of $300 \mathrm{~Hz}$ installed at 12 points on the front $\left(f_{n}\right)$ and rear screed $\left(r_{n}\right)$, as shown in Fig. 1. The excitation of the tamper and screed are defined by the ratios of $\kappa$ and $\delta$ as follows:

$$
\begin{aligned}
& \kappa=\frac{\omega_{t}}{\omega_{t \max }}=\frac{f_{t}}{f_{t \max }} \\
& \delta=\frac{\omega_{s}}{\omega_{s \max }}=\frac{f_{s}}{f_{s \max }}
\end{aligned}
$$

where $\left\{\omega_{t}, \omega_{s}\right\}$ and $\left\{\omega_{\text {tmax }}, \omega_{\text {smax }}\right\}$ are the rotation angular velocities and maximum rotation angular velocities of tamper and vibrator screed; $\left\{f_{t}, f_{s}\right\}$ and $\left\{f_{\text {tmax }}, f_{\text {smax }}\right\}$ are their corresponding excitation frequencies, $\left(f_{\text {tmax }}=20.83\right.$ and $f_{\text {smax }}=45 \mathrm{~Hz}$ ).

A multi-point measurement method of vertical RMS accelerations under the screed floor has been applied and performed in two cases: (1) under excitation frequencies of tamper $f_{t}=[10 \%, 20 \%, \ldots, 100 \%] \times f_{\text {tmax }}$ corresponding to $\kappa=[0.1,0.2, \ldots, 1.0]$ and without the excitation of vibrator screed $\delta=0$. (2), also under the same excitations of $\kappa$ but adding $50 \%$ of the maximum excitation of vibrator screed $\delta=0.5$. Through the signal processor, the measured data of the acceleration responses and their RMS values at 24-test positions have been displayed.

This experimental method can simultaneously determine the acceleration responses at different points under the front/rear screed floors, while previous studies have only measured the acceleration response at each measurement point ${ }^{3}$ or at three different points under the screed floor. ${ }^{17}$ Therefore, this 


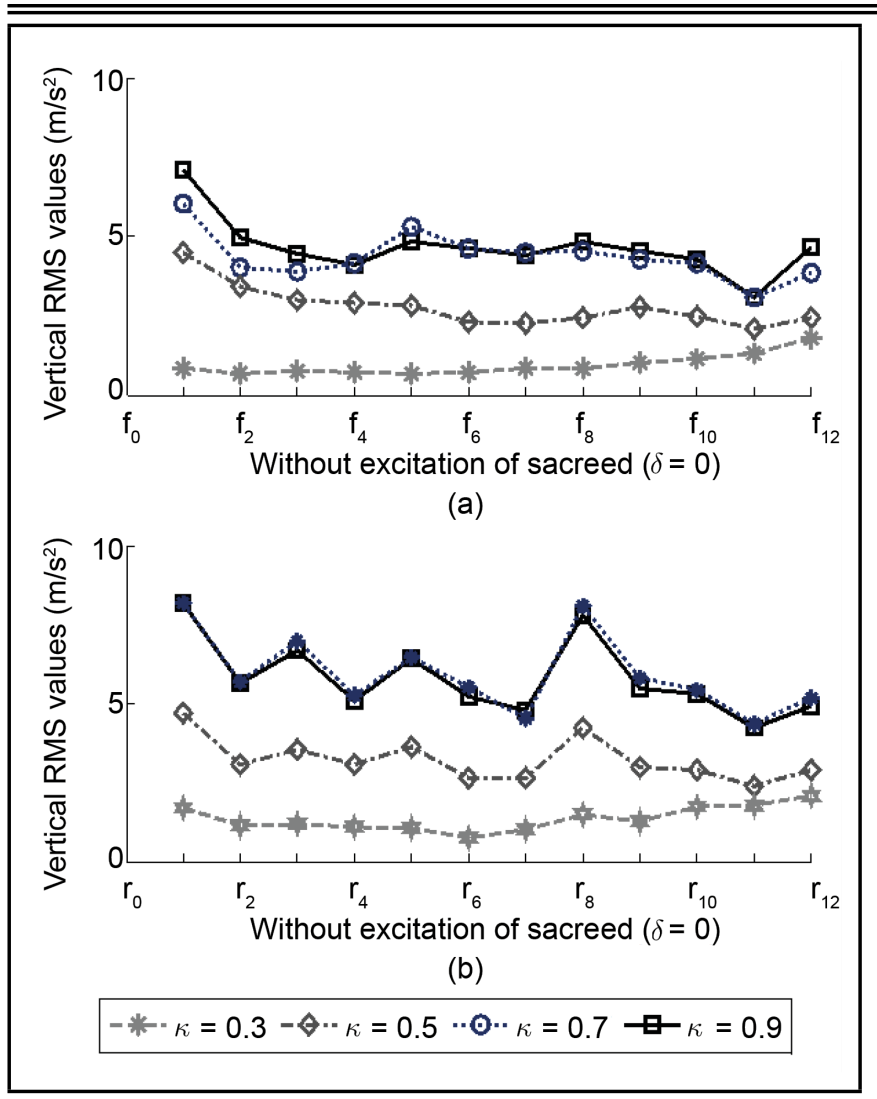

Figure 2. Measured RMS results at the front (a) and rear screeds (b) without excitation of the vibrator screed.

method easily analyzes the stability and compressive efficiency as well as calculates the vibration shaking of the VSS in comparison with the previous experiments.

\subsection{Analysis of Measurement Results}

The measured RMS results under the front/rear screed floors at the different excitations of the tamper $\kappa=[0.3,0.5,0.7,0.9]$ with $\delta=[0,0.5]$ are shown in Figs. 2 and 3. Without the excitation of the vibrator screed $\delta=0$, observing Figs. 2a and $2 b$, the RMS values at the measured points under the front/rear screed floors are relatively uniform and symmetrical with $\kappa=[0.3,0.5]$. It implies that the paving quality is relatively stable, but the compression efficiency is low due to the RMS values being quite small. With $\kappa$ is 0.7 or 0.9 , the RMS values are strongly increased, thus, the compression efficiency of the VSS is also enhanced. However, the RMS values at the measured points are remarkably skewed and asymmetrical, so the VSS works are unstable and the paving quality is low.

Under the actual paving condition of the AP, the excitation of the vibrator screed $f_{s}$ has been added to the VSS part from the main excitation $f_{t}$ of the tamper. With adding $50 \%$ the excitation of $f_{s}(\delta=0.5)$, the results in Figs. 3a and 3b show that the RMS values at the measured points are not only greatly skewed but also higher than their results without the excitation of $f_{s}$, especially at $\kappa=0.3$. This issue is due to the influence of the vibration excitation of $f_{s}$ apart from the main excitation of $f_{t}$. Similarly to the case without the excitation of the vibrator screed, the compression efficiency of the VSS is also increased while the paving quality and stability are reduced.

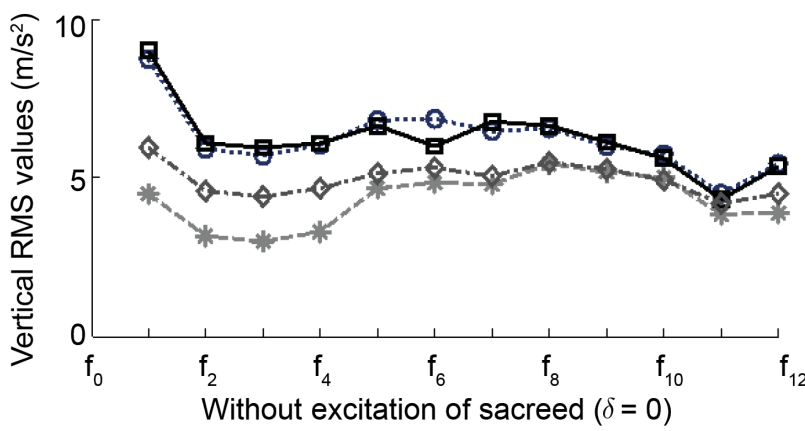

(a)

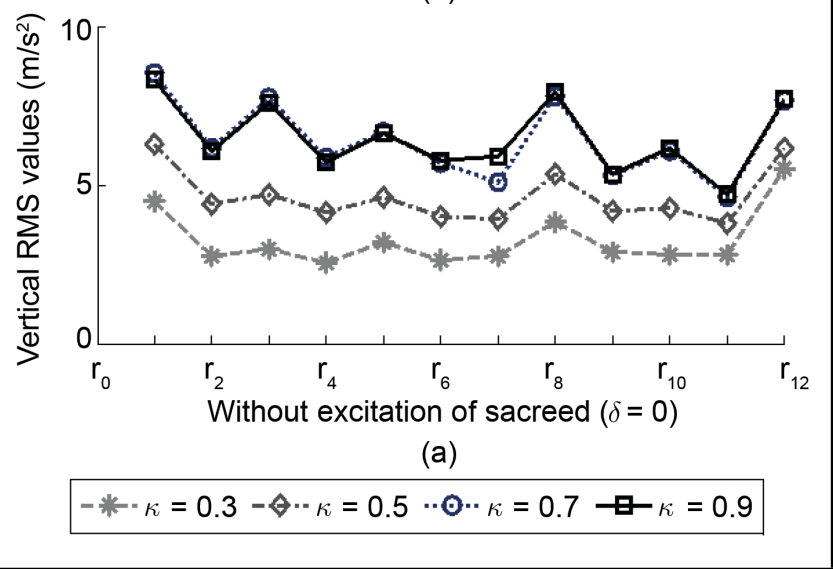

Figure 3. Measured RMS results at the front (a) and rear screeds (b) with excitation of the vibrator screed.

Consequently, it can be concluded that both vibration excitations of $f_{t}$ and $f_{s}$ greatly affect the paving performance of the AP.

Based on the experimental results, the RMS values at the points on the screed floor are greatly different. It means that the screed shaking is large under the excitation frequencies of $f_{t}$ and $f_{s}$. However, this issue has not yet been concerned in the previous studies. To determine the pitching and rolling vibrations of the screed, the RMS acceleration response of the vertical, pitching and rolling vibrations at the centre of gravity of the screed has been calculated based on the RMS value at the measured points and kinetic relationship of the screed. Assuming that the angular deformations of the screed are negligible, the RMS values at the centre of gravity of screed have been determined by:

$$
\begin{aligned}
& \ddot{z}=\frac{\ddot{z}_{f_{6}} b_{4}+\ddot{z}_{r_{6}} b_{3}}{b_{3}+b_{4}} ; \quad \ddot{\phi}=\frac{\ddot{z}_{r_{6}}-\ddot{z}_{f_{6}}}{b_{3}+b_{4}} ; \\
& \ddot{\theta}=\frac{\left(\ddot{z}_{r_{1}}-\ddot{z}_{r_{11}}\right) d_{3}+\left(\ddot{z}_{f_{1}}-\ddot{z}_{f_{11}}\right) d_{4}}{\left(l_{1}+l_{2}\right)\left(d_{3}+d_{4}\right)} ;
\end{aligned}
$$

where $\ddot{z}_{k}$ is the vertical RMS acceleration at the measured points of $k, k=f_{1}, f_{6}, f_{11}, r_{1}, r_{6}, r_{11} ; b_{3,4}$ and $l_{1,2}$ are the distances from the centre of gravity of screed to the corresponding measurement points in the axis of $x$ and $y$.

The measured RMS results at the centre of gravity of screed under the excitations of $\kappa=[0.1,0.2, \ldots, 1.0]$ with $\delta=$ $[0,0.5]$ are plotted in Figs. $4 \mathrm{a}-4 \mathrm{c}$. The results show that the vertical, pitching and rolling RMS values of the screed are greatly affected by both ratios of $\kappa$ and $\delta$, especially the ratio of 


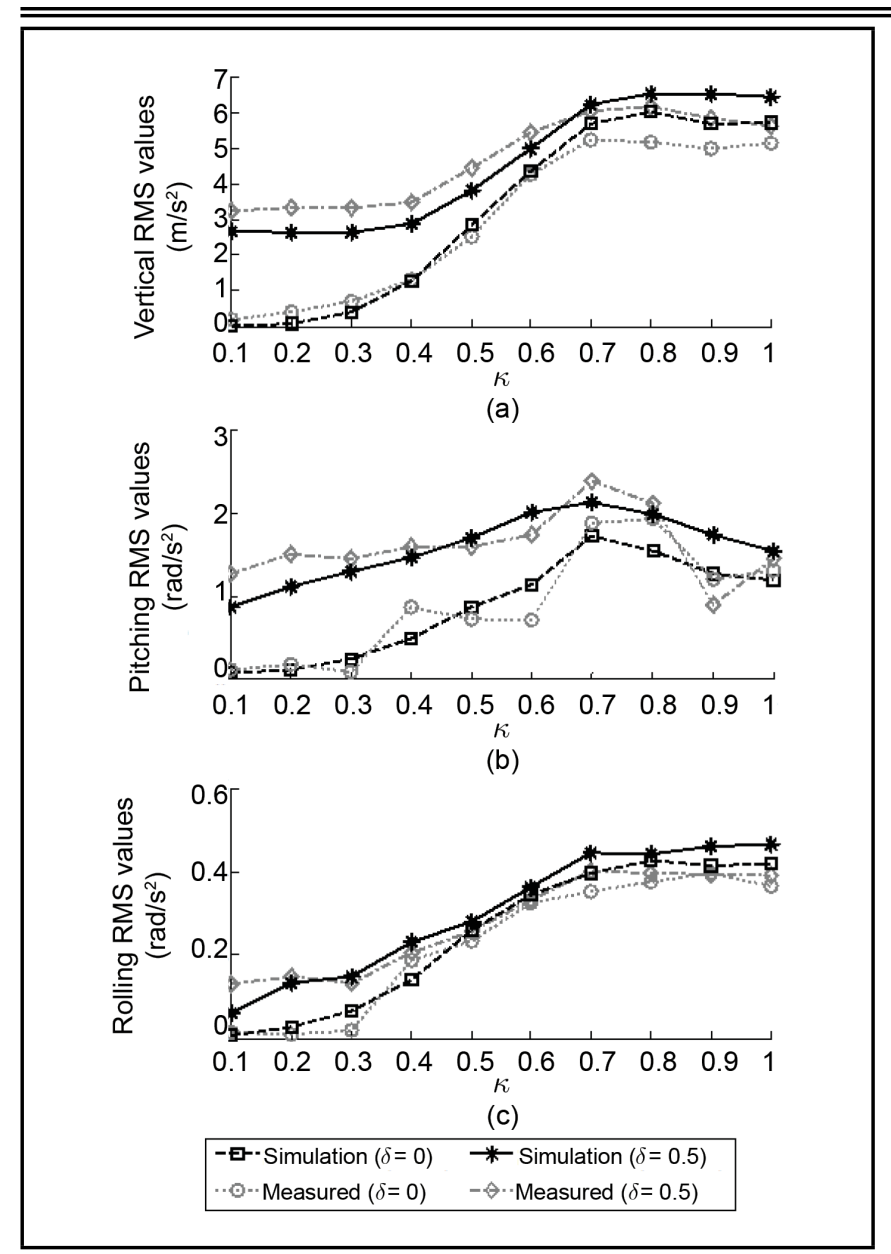

Figure 4. RMS results of vertical (a), pitching (b), and rolling vibrations (c) at the center of gravity of the screed.

$\kappa$. The RMS values are slightly increased with $0.1 \leq \kappa \leq 0.5$ and quickly enhanced with $0.5 \leq \kappa \leq 0.8$ corresponding the excitation frequency of $10.4 \leq f_{t} \leq 16.7 \mathrm{~Hz}$. Thus, the compression efficiency of the VSS is maximized in this frequency range. However, the RMS values are insignificantly increased with $0.8 \leq \kappa \leq 1.0$, this can be due to the influence of the frictional resistance in the VSS. Thus, to increase the compression efficiency, the excitation frequency of the tamper should be chosen by $10.4 \leq f_{t} \leq 16.7 \mathrm{~Hz}$, especially at $F_{t}=14.6 \mathrm{~Hz}$ $(\kappa=0.7)$. This analysis result is also close to the result in Ref. ${ }^{6}$ However, the paving quality is significantly reduced due to increasing the maximum screed shaking.

The paving performance of the AP has been affected by the VSS's parameters. ${ }^{1,4}$ However, it is difficult to evaluate the influence of parameters via experimentation. To solve this problem, a 3D nonlinear dynamic model of the VSS-AP is built to analyze the vibration of the VSS.

\section{MODELLING OF THE VSS}

\subsection{Mathematical Model}

Based on the actual structure of the VSS-AP, a 3D nonlinear dynamic model which can fully reflect the screed shaking has been established as in Fig. 5 .

In Fig. 5, the vertical, pitching, and rolling motions of screed are defined by $z, \phi$, and $\theta$, respectively; the mass of screed, first and second tampers is described as $m, m_{f i}$ and $m_{r i}$; the stiffness and damping coefficients of pavement at the first/second tamper and screed are symbolized by $k_{t i}, k_{r i}, k_{n i}$ and $c_{t i}, c_{r i}, c_{n i} ; b_{n}$ and $l_{1-2}$ are the longitudinal and lateral distances, $i=1,2, \ldots, 8, n=1,2,3,4)$.

According to the vibration theory and the VSS-AP model, the vibration equations have been expressed as:

$$
\begin{aligned}
& m \ddot{z}=-\sum_{n=1}^{4} F_{n}+\sum_{i=1}^{8} F_{t i}+F_{s} ; \\
& I_{y} \ddot{\phi}=\sum_{n=1}^{4}(-1)^{n+1} F_{n} b_{v}+\sum_{i=1}^{8} F_{t i} b_{2}-F_{s} b_{1} ; \\
& I_{x} \ddot{\theta}=\sum_{n=1}^{4}(-1)^{u} F_{n} l_{u}+\sum_{i=1}^{4} F_{t i} l_{t i}-\sum_{i=4}^{8} F_{t i} l_{t i} ;
\end{aligned}
$$

where $F_{n}$ is the vertical dynamic force of the vibrator screedpavement interaction in the paving process; $F_{t i}$ and $F_{s}$ are the excitation forces of tamper and vibrator screed.

The vertical dynamic force $F_{n}$ has been determined by:

$$
\begin{aligned}
& F_{n}=k_{n}\left[z+(-1)^{n} b_{v} \phi+(-1)^{u+1} l_{u} \theta+\right. \\
& c_{n}\left[\dot{z}+(-1)^{n} b_{v} \dot{\phi}+(-1)^{u+1} l_{u} \dot{\theta}\right] ;
\end{aligned}
$$

where $\left\{\begin{array}{l}n=1,2 \\ n=3,4\end{array}\right.$ then $\left\{\begin{array}{l}u=1 \\ u=2\end{array}\right.$ and $\left\{\begin{array}{l}v=n+2 \\ v=n\end{array}\right.$.

The excitation force $F_{t i}$ has been calculated based on the VSS-AP model in Fig. 5b as follows:

$$
\begin{aligned}
& F_{t i}=\sum_{x=f, r}\left(-m_{x i} \ddot{z}_{x i}+c_{x i} \dot{z}_{x i}+k_{x i} z_{x i}\right) ; \\
& z_{x i}=e_{x i} \sin \left(\omega_{t} t+\beta_{i}+\psi\right) ; \psi=a \alpha+b \gamma ;
\end{aligned}
$$

where $\left\{\begin{array}{l}i=1,2,3,4 \\ i=5,6,7,8\end{array}\right.$ then $\left\{\begin{array}{l}a=0 \\ a=1\end{array} ;\left\{\begin{array}{l}x=f \\ x=r\end{array}\right.\right.$ then $\left\{\begin{array}{l}b=0 \\ b=1\end{array} ; \alpha, \beta_{i}\right.$, and $\gamma$ are the angular deviations of excitation forces between the right and left sides, between tampers, and between the first/second tampers of the tamper mechanisms, respectively.

By replacing Eq. (6) into (5) and mathematical transformation, we have:

$$
F_{t i}=\sum_{x=f}^{r}\left\{\begin{array}{l}
m_{x i} e_{x i} \omega_{t}^{2} \sin \left(\omega_{t} t+\beta_{i}+\psi\right)+ \\
+c_{x i} e_{x i} \omega_{t} \cos \left(\omega_{t} t+\beta_{i}+\psi\right)+ \\
+k_{x i} e_{x i} \sin \left(\omega_{t} t+\beta_{i}+\psi\right)
\end{array}\right\}
$$

The excitation force of the vibrator screed $F_{s}$ has been determined by:

$$
F_{s}=m_{s} e_{s} \omega_{s}^{2} \sin \omega_{s} t
$$

where $m_{s}$ and $e_{s}$ are the mass and distance of the eccentric configuration.

By combining Eqs. (3), (4), (7) and (8), the vertical, pitching, and rolling accelerations at the center of the screed have then been determined. 


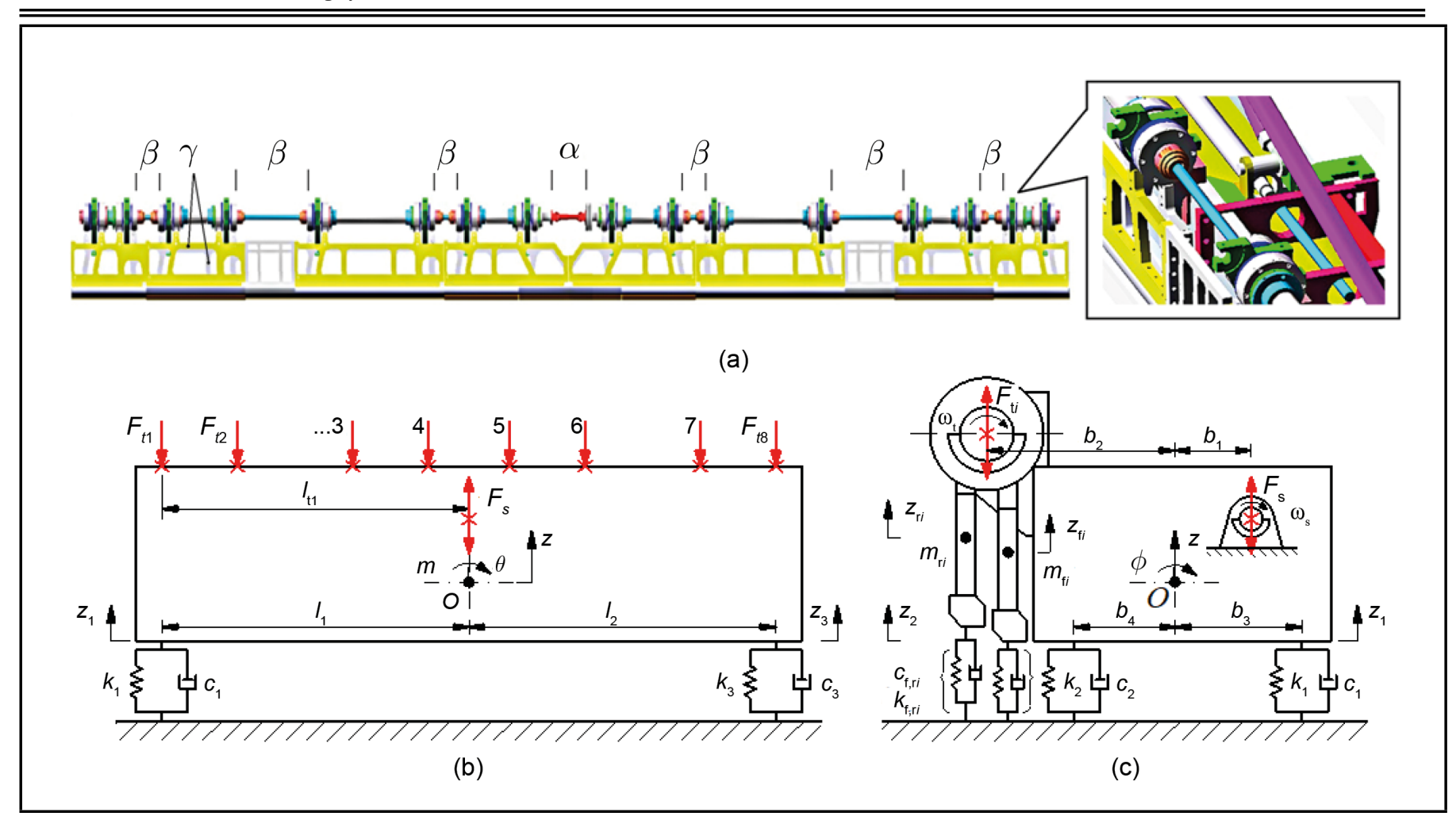

Figure 5. The structure and angular deviations $\alpha, \beta, \gamma$ of tamper (a), 3D dynamic model of the VSS-AP with the front of the screed (b) and the side of the screed (c).

Also, the vertical acceleration at points on the front/rear screed floors in Fig. 1c have been given by:

$$
\ddot{z}_{\chi}=\ddot{z}+(-1)^{v} b_{v+2} \ddot{\phi}+(-1)^{v+1} l_{y} \ddot{\theta}
$$

where subscript $\chi$ denotes $f_{y}$ or $r_{y}$; when $y=0,1, \ldots, 6$ then $v=1$, and when $y=7,8, \ldots, 12$ then $v=2$.

To evaluate the influence of the vibration on the systems, the RMS acceleration response was used as the evaluation index. $^{24,25}$ In this study, the paving performance of the AP is evaluated via the RMS values at points on the screed floor or the centre of gravity of screed as follows:

$$
\begin{aligned}
& R M S_{\chi}=\left[\frac{1}{T} \int_{0}^{T}\left\{\ddot{z}_{\chi}(t)\right\}^{2} d t\right]^{1 / 2} ; \\
& R M S_{w}=\left[\frac{1}{T} \int_{0}^{T}\left\{a_{w}(t)\right\}^{2} d t\right]^{1 / 2} ;
\end{aligned}
$$

where $\ddot{z}_{\chi}(t)$ is the vertical acceleration responses calculated by Eq. (9); subscript $w$ refers to the vertical, pitching, and rolling motions at the centre of gravity of screed; $a_{w}(t)$ is the acceleration at the motion of $w$; and $T$ is the duration of the simulation.

Therefore, the result of the $\mathrm{RMS}_{\kappa}$ values on the screed floor is high and uniform; or the result of the $\mathrm{RMS}_{z}$ value is high and both the $\mathrm{RMS}_{\phi}$ and $\mathrm{RMS}_{\omega}$ values are low, it means that the compression efficiency, paving quality, and working stability of the VSS are better.

\subsection{Influence of the Parameters of the VSS}

To analyze the influence of the dynamic parameters of the VSS on the paving performance based on the dynamic model
Table 1. The numerical values of the VSS-AP.

\begin{tabular}{||c|c|c|c||}
\hline Parameter & Value & Parameter & Value \\
\hline$m / \mathrm{kg}$ & 3083 & $e_{f i} / \mathrm{m}$ & $3 \times 10^{-3}$ \\
\hline$m_{f 1, f 8} / \mathrm{kg}$ & 78 & $e_{r i} / \mathrm{m}$ & $3 \times 10^{-3}$ \\
\hline$m_{f 2, f 7} / \mathrm{kg}$ & 110 & $\omega_{\text {tmax }} / \mathrm{rpm}$ & 1250 \\
\hline$m_{f 3, f 6} / \mathrm{kg}$ & 70 & $\omega_{\text {smax }} / \mathrm{rpm}$ & 2700 \\
\hline$m_{f 4, f 5} / \mathrm{kg}$ & 69 & $f_{\text {tmax }} / \mathrm{Hz}$ & 20.83 \\
\hline$m_{r 1, r 8} / \mathrm{kg}$ & 80 & $f_{s m a x} / \mathrm{Hz}$ & 45 \\
\hline$m_{r 2, r 7} / \mathrm{kg}$ & 114 & $k_{1,3} / \mathrm{N} \mathrm{m}^{-1}$ & $3.57 \times 10^{6}$ \\
\hline$m_{r 3, r 6} / \mathrm{kg}$ & 71 & $k_{2,4} / \mathrm{N} \mathrm{m}^{-1}$ & $3.06 \times 10^{6}$ \\
\hline$m_{r 4, r 5} / \mathrm{kg}$ & 72 & $k_{f, r, i} / \mathrm{N} \mathrm{m}^{-1}$ & 0 \\
\hline$b_{1} / \mathrm{m}$ & 0.114 & $c_{1,3} / \mathrm{Ns} \mathrm{m}^{-1}$ & $58 \times 10^{3}$ \\
\hline$b_{2} / \mathrm{m}$ & 0.2 & $c_{2,4} / \mathrm{Ns} \mathrm{m}^{-1}$ & $68 \times 10^{3}$ \\
\hline$b_{3} / \mathrm{m}$ & 0.332 & $c_{f, r, i} / \mathrm{Ns} \mathrm{m}^{-1}$ & 0 \\
\hline$b_{4} / \mathrm{m}$ & 0.163 & $\alpha /{ }^{\circ}$ & 60 \\
\hline$l_{1} / \mathrm{m}$ & 6 & $\beta /{ }^{\circ}$ & 90 \\
\hline$l_{2} / \mathrm{m}$ & 6 & $\gamma /{ }^{\circ}$ & 90 \\
\hline$e_{s} / \mathrm{m}$ & $2.5 \times 10^{-3}$ & $i=1,2, \ldots, 12$ & \\
\hline
\end{tabular}

of the VSS-AP, the accuracy of the mathematical model should be verified by comparing the results between simulation and experiment methods under the same operating conditions of $\kappa=[0.1,0.2, \ldots, 1.0]$ with $\delta=[0,0.5]$. With the reference parameters of the VSS-AP, as listed in Table 1, the simulation results of RMS values at the centre of gravity of screed are compared with the measured results in the same Figs. $4 \mathrm{a}-4 \mathrm{c}$. Observing the comparison results, it can see that the characteristic curves between simulation and measurement results are similar. Thus, VSS-AP's mathematical model can be reliable in analysis of the influence of dynamic parameters.

The VSS's vibration is greatly affected at $\kappa=0.7$. Thus, to evaluate the influence of other dynamic parameters 


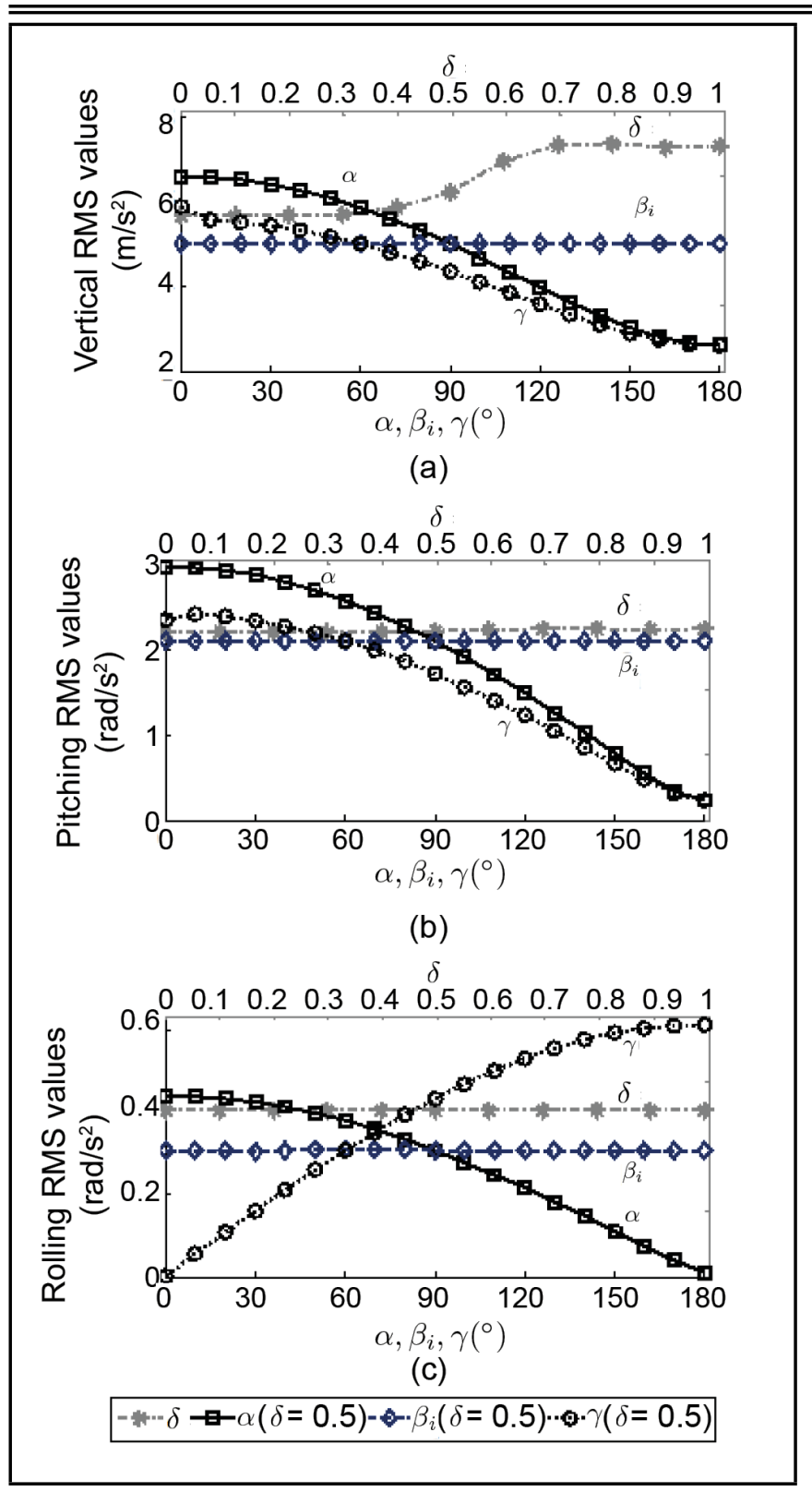

Figure 6. Influence of parameters of the VSS at $\kappa=0.7$.

$\left\{\delta, \alpha, \beta_{i}, \gamma\right\}$, the numerical values of $\delta=[0.1,0.2, \ldots, 1.0]$ and of $\left\{\alpha, \beta_{i}, \gamma\right\}$ from $0^{\circ}$ to $180^{\circ}$ are respectively simulated under the working condition of $\kappa=0.7$. The RMS results of the screed are shown in Figs. 6a- 6c.

Influence of the ratio $\delta$ : Observing Fig. 6a, the vertical RMS value is lightly changed at $0 \leq \delta<0.5$ and $0.8<\delta \leq$ 1.0. It is quickly increased at $0.5 \leq \delta \leq 0.8$ corresponding the excitation frequency of $22.4 \leq f_{s} \leq 36 \mathrm{~Hz}$. However, both pitching and rolling RMS values are insignificantly changed, as shown in Figs. $6 \mathrm{~b}$ and $6 \mathrm{c}$. This is due to the mass of eccentric configuration of the vibrator screed being installed at the centre of gravity of screed. Therefore, the paving performance of the AP can be improved by control of the excitation frequency of $22.4 \leq f_{s} \leq 36 \mathrm{~Hz}$, especially at $f_{s}=31.5 \mathrm{~Hz}$ $(\delta=0.7)$.

Influence of the angular deviation $\alpha$ : Also observing Figs. $6 a-6 c$, all the vertical, pitching and rolling RMS values of the screed are quickly reduced when the angular deviation $\alpha$ increases from $0^{\circ}$ to $180^{\circ}$. The RMS values of the screed are maximum at $\alpha=0^{\circ}$ due to the remote ends of the VSS not vibrating out of phase, thus the compression efficiency of the screed is maximum, while the paving quality and working stability are minimum due to increasing the screed shaking. Contrariwise, the RMS values are minimum at $\alpha=180^{\circ}$, therefore, the compression efficiency of the screed is the smallest. To improve the paving performance of the AP, the angular deviation $\alpha$ should be controlled in a range of $30 \circ$ to 120 .

Influence of the angular deviation $\beta_{i}$ : The RMS results are also given in the same Figs. $6 a-6 c$. It can see that the RMS values of the screed are insignificantly affected by $\beta_{i}$. This is due to the angular deviations $\beta_{i}$ between excitation forces of tampers being symmetrically designed following the tamper mechanisms.

Influence of the angular deviation $\gamma$ : The simulation results are also plotted in Fig. 6. Observing both Figs. 6a and 6b, we can see that both the vertical and pitching RMS values of the screed are decreased with increasing the angular deviation of $\gamma$ from $0^{\circ}$ to $180 \circ$, while the rolling RMS value is increased, as shown in Fig. 6c. Both the vertical and pitching RMS values can reach a maximum at a range of $\gamma$ from $0^{\circ}$ to $60^{\circ}$. Therefore, the compression efficiency of the VSS is the maximum. With the width of $12000 \mathrm{~mm}$ of the screed, the rolling vibration thus strongly influences the paving quality and working stability of the VSS-AP. However, the rolling RMS value is the smallest in a range of $\gamma$ from $0^{\circ}$ to $60^{\circ}$. Accordingly, to improve the paving performance, the angular deviation of $\gamma$ from $0^{\circ}$ to $60^{\circ}$ should be controlled.

Based on the above analysis results, it is deduced that three dynamic parameters of $\delta, \alpha$, and $\gamma$ greatly influence the paving performance of the AP apart from the main excitation of $f_{t}$. The compression efficiency of the VSS is increased while both paving quality and working stability are reduced and vice versa. It is very difficult to satisfy all objective functions simultaneously. In addition, only excitation frequencies of $f_{t}$ and $f_{s}$ have been optimized, ${ }^{1,2,4,18}$ the influence of the angular deviations of the tamper has not yet been concerned in the previous researches. Moreover, the excitation frequencies of $f_{t}$ and $f_{s}$ are always changed and depended on the operator's subjectivity. Consequently, to improve the paving performance of the AP, the angular deviations of $\alpha$ and $\gamma$ of the tamper should be controlled based on the change of excitation frequencies and screed shaking accelerations.

\section{CONTROL VIBRATION OF THE VSS}

\subsection{Control Model for the VSS-AP}

The vibration excitation forces $F_{t i}$ of the tamper depend on the angular deviations of $\alpha$ and $\gamma$, as described in Eqs. (6) and (7). To control the vibration of the VSS, fuzzy logic control (FLC) is applied to determine the optimal values of $\alpha$ and $\gamma$ based on two output signals of the pitching and rolling accelerations of the screed and an input signal of the excitation frequency $f_{t}$. The control model is designed as in Fig. 7 . 


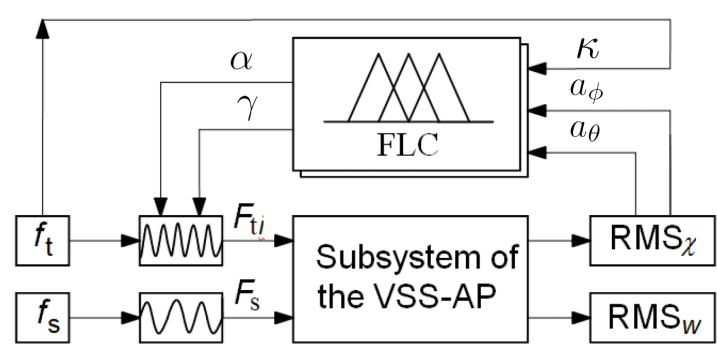

Figure 7. Control system model of the VSS-AP.

Based on the characteristic curves of $\kappa, \alpha$, and $\gamma$ in Figs. 4 and 6 , and analysis results of the influence of parameters on paving performance of the AP, the numerical values $\alpha$ and $\gamma$ of the control function in Eq. (6) $\psi=a \alpha+b \gamma$ have been expressed as:

$$
\begin{cases}30^{\circ} \leq \alpha \leq 90^{\circ} ; 0^{\circ} \leq \gamma \leq 60^{\circ} ; & \text { if } \kappa \leq 0.7 \\ 60^{\circ} \leq \alpha \leq 120^{\circ} ; 30^{\circ} \leq \gamma \leq 90^{\circ} ; & \text { else }\end{cases}
$$

The numerical values of $\alpha$ and $\gamma$ in Eq. (12) are then controlled based on the FLC.

Appling the FLC: In order to control the vibration systems, the control methods as PID, FLC, or H were mainly applied. Further development was the combination of control methods such as PID-Fuzzy, PID-Neural, Skyhook-Fuzzy. ${ }^{25-28}$ The different control methods were applied depending on different control objectives. This research aim is to control numerical values of $\alpha$ and $\gamma$ to reach the goals of the maximum $\mathrm{RMS}_{z}$ value, minimum $\mathrm{RMS}_{\phi}$ and $\mathrm{RMS}_{\gamma}$ values. In the above control methods, the FLC is a controller that does not depend on the designed operation conditions, on the contrary, the FLC can control multi-objective based on its wide and fuzzy inference system. ${ }^{25,29}$ Thus, the FLC is suitable for controlling the vibration of the VSS.

FLC's design process: The structure of FLC includes a fuzzification interface (FI), a fuzzy inference system (FIS), and a defuzzification interface (DI). The control principle of the FLC is that the input-numerical values in FI are firstly transformed into input-linguistic variables (LVs), the FIS is then used to infer output-LVs from input-LVs based on the membership function of control rules, and finally, the output-LVs are transformed back to output-numerical values via DI. ${ }^{30}$

Based on the FLC model in Fig. 7, three input-numerical values $\left\{\kappa, a_{\phi}, a_{\theta}\right\}$ and two output-numerical values $\{\alpha, \gamma\}$ with their membership functions are defined as in Fig. 8. Herein, three input-LVs are defined by minimum (MIN, $\kappa \leq$ 0.7 ), maximum (MAX, $\kappa>0.7$ ), negative big (NB), negative medium (NM), negative small (NS), zero (Z), small (S), medium (M), and big (B). Besides, two output-LVs are also defined as very small (VS), small (S), small medium (SM), medium (M), medium big (MB), and big (B), respectively.

If-then rules in FIS are applied to describe the relationship of input- and output-numerical values based on the analysis results in Figs. 4 and 6 and the designer's experience. There

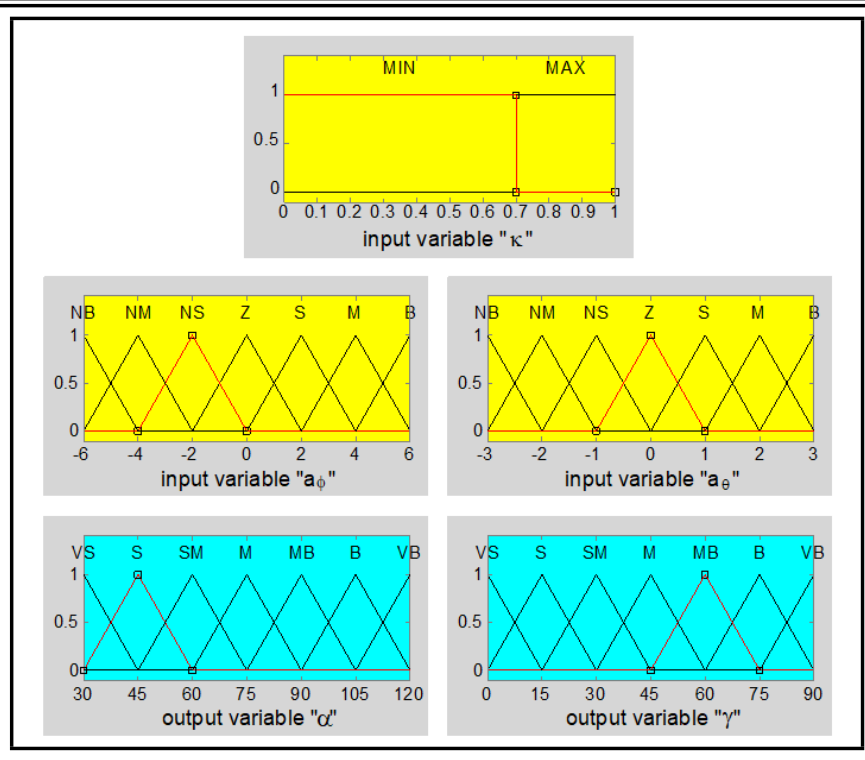

Figure 8. Input-output variables of the membership functions.

Table 2. The control rules of FIS with $\kappa \leq 0.7$

\begin{tabular}{|c|c|c|c|c|c|c|c|c|}
\hline \multicolumn{2}{|c|}{$\kappa=\mathrm{MIN}$} & \multicolumn{7}{|c|}{$a$} \\
\hline & & NB & NM & NS & $\mathrm{Z}$ & S & M & B \\
\hline \multirow{7}{*}{$a$} & NB & MB & MB & M & M & M & MB & MB \\
\hline & NM & MB & M & SM & $\mathrm{S}$ & SM & M & $\mathrm{MB}$ \\
\hline & NS & M & SM & $\mathrm{S}$ & VS & S & SM & M \\
\hline & $\mathrm{Z}$ & SM & S & VS & VS & VS & S & SM \\
\hline & S & M & SM & S & VS & $S$ & SM & M \\
\hline & M & MB & M & SM & $S$ & SM & M & MB \\
\hline & B & MB & MB & M & M & M & MB & MB \\
\hline
\end{tabular}

Table 3. The control rules of FIS with $\kappa>0.7$.

\begin{tabular}{|c|c|c|c|c|c|c|c|c|}
\hline \multirow{2}{*}{\multicolumn{2}{|c|}{$\kappa=$ MAX }} & \multicolumn{7}{|c|}{$a$} \\
\hline & & NB & $\mathrm{NM}$ & NS & $\mathrm{Z}$ & $S$ & $M$ & B \\
\hline \multirow{7}{*}{$a$} & NB & VB & VB & B & B & B & VB & VB \\
\hline & $\mathrm{NM}$ & VB & B & MB & $M$ & MB & B & VB \\
\hline & NS & B & MB & $\mathrm{M}$ & SM & $\mathrm{M}$ & $\mathrm{MB}$ & B \\
\hline & $\mathrm{Z}$ & $\mathrm{MB}$ & M & SM & SM & SM & M & $\mathrm{MB}$ \\
\hline & S & B & MB & $\mathrm{M}$ & SM & $\mathrm{M}$ & MB & B \\
\hline & $\mathrm{M}$ & VB & B & MB & $\mathrm{M}$ & MB & B & VB \\
\hline & B & VB & VB & B & B & B & VB & VB \\
\hline
\end{tabular}

are ninety-eight rules in which forty-nine rules are given in Table 2 and forty-nine rules are given in Table 3 as follows, $(i=1-49)$ :

If $\kappa$ is MIN, $a_{\phi}$ is $A_{i}$, and $a_{\theta}$ is $B_{i}$, then $\alpha$ and $\gamma$ are $C_{i}$; If $\kappa$ is MAX, $a_{\phi}$ is $A_{i}$, and $a_{\theta}$ is $B_{i}$, then $\alpha$ and $\gamma$ are $D_{i}$.

According to the minimum function and the centroid method of Mamdani and Assilian, ${ }^{29}$ the FIS of Mamdani has been selected to control the VSS model.

\subsection{Control Results}

Based on the control system model and the FLC method, the numerical simulation is then performed to control the vibration of the VSS. The control results of the acceleration responses at the centre of gravity of screed with $\kappa=\delta=0.7$ are plotted in Fig. 9.

The control results show that the vertical acceleration re- 


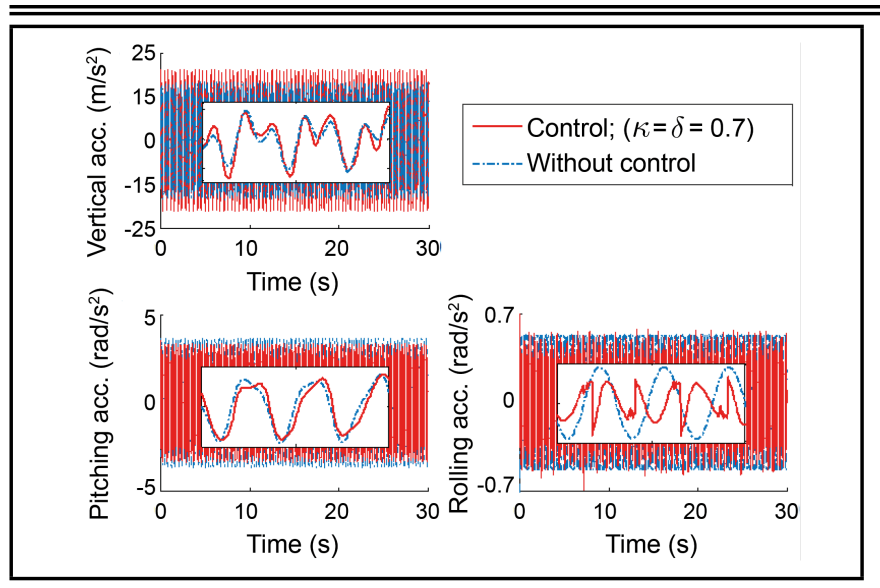

Figure 9. Acceleration responses of the screed with $\kappa=\delta=0.7$.

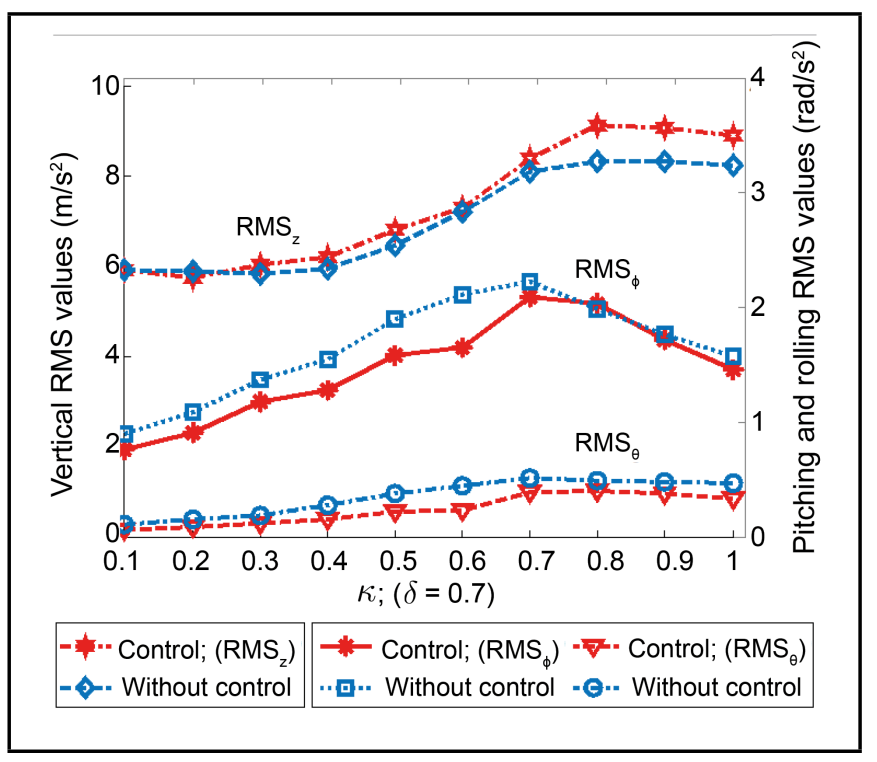

Figure 10. Control results of the vertical, pitching, and rolling RMS accelerations at the center of gravity of the screed.

sponse is higher while both the pitching and rolling acceleration responses are smaller their results without control. This means that the compression efficiency, paving quality, and working stability are improved with the controlled parameter. Additionally, the paving performance with control is also evaluated under different ratios of $\kappa$ with $\delta=0.7$, as shown in Fig. 10. Observing Fig. 10, the control results show that the vertical RMS value is increased while both the pitching and rolling RMS values are also decreased in comparison with their RMS results without control. It implies that the paving performance of the AP is significantly improved under different excitation frequencies $f_{t}$ of tamper.

With the width of $12000 \mathrm{~mm}$ of the screed, the rolling acceleration of screed greatly affects paving quality and working stability. Therefore, the vertical RMS accelerations at points on the front/rear screed floors are also used to evaluate the control performance on the paving quality and working stability of the VSS under two different working conditions of $\kappa=\delta=0.5$ and $\kappa=\delta=0.7$. The control results of RMS values are plotted in Fig. 11, and their maximum (Max-) and minimum (Min-) RMS values are also listed in Tables 4 and 5.
Table 4. Maximum and Minimum RMS values on the front screed floor.

\begin{tabular}{|c|c|c|c|c|}
\hline \multicolumn{2}{|c|}{ RMS values/m.s } & Max-RMS & Min-RMS & Deviation \\
\hline$\kappa=\delta=0.5$ & $(1)$ & 5.313 & 5.195 & $2.22 \%$ \\
\cline { 2 - 5 } & $(2)$ & 5.316 & 4.886 & $8.09 \%$ \\
\hline \multirow{2}{*}{$\kappa=\delta=0.7$} & $(1)$ & 8.657 & 8.529 & $1.48 \%$ \\
\cline { 2 - 5 } & $(2)$ & 8.978 & 8.226 & $8.38 \%$ \\
\hline
\end{tabular}

(1) Control (2) Without control

Table 5. Maximum and Minimum RMS values on the rear screed floor.

\begin{tabular}{|c|c|c|c|c|}
\hline \multicolumn{2}{|c|}{ RMS values/m.s } & Max-RMS & Min-RMS & Deviation \\
\hline$\kappa=\delta=0.5$ & $(1)$ & 4.953 & 4.886 & $1.35 \%$ \\
\cline { 2 - 5 } & $(2)$ & 5.028 & 4.456 & $11.38 \%$ \\
\hline$\kappa=\delta=0.7$ & $(1)$ & 8.402 & 8.315 & $1.04 \%$ \\
\cline { 2 - 5 } & $(2)$ & 9.030 & 7.822 & $13.38 \%$ \\
\hline
\end{tabular}

(1) Control (2) Without control

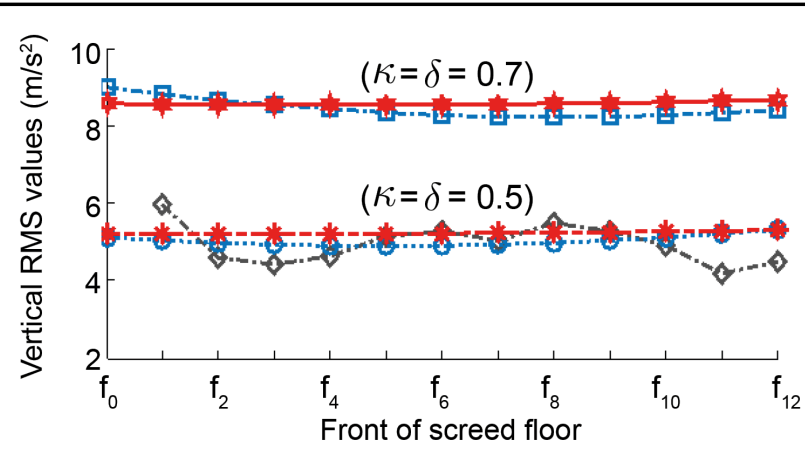

(a)

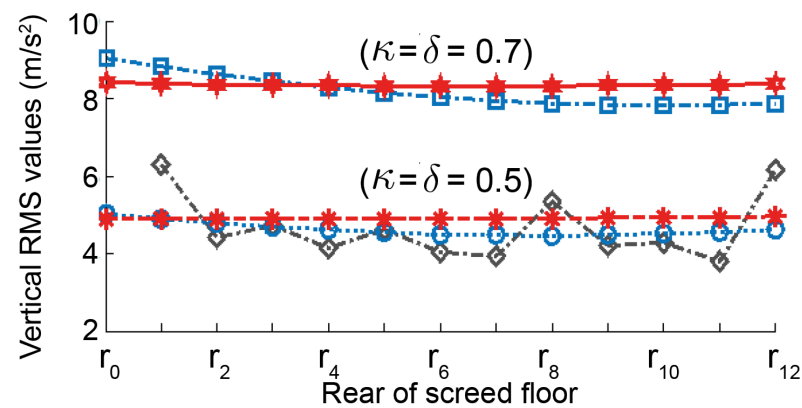

(b)

\begin{tabular}{|c|c|}
\hline $\begin{array}{l}-*-\text { Control; }(\kappa=\delta=0.5) \\
-\circ \cdot-\text { Without Control } \\
-\diamond \cdot-\text { Measured; }(\kappa=\delta=0.5\end{array}$ & $\begin{array}{l}- \text { Control; }(\kappa=\delta=0.7) \\
-\square-\text { Without Control }\end{array}$ \\
\hline
\end{tabular}

Figure 11. Control result of the RMS values at the front (a) and rear screeds (b).

Observing Fig. 11a, under both two excitation cases of $\kappa=\delta=0.5$ and $\kappa=\delta=0.7$, the vertical RMS values with control at points on the front screed floor are relatively uniform in comparison with their values without control. Besides, the Max- and Min-RMS values on the front screed floor in Table 4 also show that the deviations between Max- and MinRMS values with control are smaller by $2.22 \%$ and $1.48 \%$, while their deviations without control are $8.09 \%$ and $8.38 \%$ under both two excitation cases. Similarly, the vertical RMS values at points on the rear screed floor with control are also more uniform than without control, as shown in Fig. 11b. The results in Table 5 also show that the deviations between Max- 
and Min-RMS values with control are smaller by $1.35 \%$ and $1.04 \%$, while their deviations without control are $11.38 \%$ and $13.38 \%$ under both two excitation cases. Consequently, it can be concluded that the paving quality and working stability of the VSS are significantly improved with the controlled parameters.

\section{CONCLUSIONS}

The paving performance of the AP is studied via an experimental method. The influence of the dynamic parameters on the paving performance is evaluated via the numerical simulation method. The numerical values of $\alpha$ and $\gamma$ are then controlled to improve the compression efficiency, paving quality, and working stability of the VSS-AP. The research results are summarized as follows:

The excitation frequencies of $f_{t}$ and $f_{s}$, and the angular deviations of $\alpha, \beta_{i}$, and $\gamma$ greatly affect the paving performance of the AP, particularly the numerical values of $f_{t}, \alpha$, and $\gamma$.

Based on the input-numerical signals of the excitation frequency $f_{t}$ and screed shacking accelerations $a_{\phi}$ and $a_{\theta}$, the compression efficiency, paving quality, and working stability of the AP are clearly improved by controlling the angular deviations of $\alpha$ and $\gamma$ under different working conditions. Especially, based on the databases of the compaction monitoring system using the global positioning system technologies, ${ }^{16}$ the fuzzy clustering techniques apply to quickly analyze the hot mix asphalt compaction data, ${ }^{10}$ or the multi-sensor infrared temperature scanning bar system used to analyze the paving quality,${ }^{17}$ the paving performance of the AP can be further improved by controlling the vibration of the VSS based on these databases.

The research results not only contribute to the existing body of knowledge on the asphalt pavers but also can provide an important reference for optimal design or control of the compression force of paving machines to further improve the paving efficiency. Besides, with the soil compactors, the excitation force of the vibratory drum is also used to compact the offroad deformable, ${ }^{19,25}$ however, the excitation force has not yet been interested and controlled. Thus, the research results can also be used as an important reference for controlling the excitation force of the vibrator drum to enhance the compaction efficiency of the soil compactors.

\section{ACKNOWLEDGEMENTS}

This work has been supported by the National Key Research and Development Plan, China (No.2019YFB2006402); Open Fund Project of Hubei Key Laboratory of Intelligent Transportation Technology and Device, Hubei Polytechnic University, China (No.2020XY105) and Talent Introduction Fund Project of Hubei Polytechnic University [No. 19XJK17R].

\section{REFERENCES}

1 Wan, Y. P. and Jia, J. Nonlinear dynamics of asphalt-screed interaction during compaction: Ap- plication to improving paving density, Construction and Building Materials, 202, 363-373, (2019). https://dx.doi.org/10.1016/j.conbuildmat.2018.12.205

${ }^{2}$ Luo, T. H., Gan, X. F. and Luo W. J. Nonlinear dynamics simulation of compacting mechanism with doubleeccentric vibrator of asphalt-paver, International Conference on Intelligent Computation Technology and Automation, (2010). https://dx.doi.org/10.1109/ICICTA.2010.155

${ }^{3}$ Luo, D., Feng, Z. X. and Wang, X. Y. Simulation and experimental study on compacting mechanism of asphalt paver, Journal of Guangxi University, 35, 729735, (2011). http://en.cnki.com.cn/Article_en/CJFDTotalGXKZ201105007.htm

${ }^{4}$ Liu, H. H., Jia, J., Ma, C. X. and Zhang Y. Investigation of paver screed on compaction characteristics of mixture, China Journal of Highway and Transport, 29, 152-157, (2016). http://zgglxb.chd.edu.cn/EN/Y2016/V29/I7/152

5 Jia, J., Wan, Y. P. and Liu, H. H. Parameter optimization for a compaction system of vibration screed of an asphalt paver based on a multi-objective genetic algorithm, Journal of vibration and shock, 36, 230-235, (2017). https://dx.doi.org/10.13465/j.cnki.jvs.2017.12.037

${ }^{6}$ Yin, C. Dynamic study and parameter optimization about screed and tamper mechanism of the paver [D]. Southeast University, (2018).

${ }^{7}$ Feng, Z. X., Zhu, L. B., Wang, X. X. and Zhao, L. J. Dynamic simulation and parametric selection for tamping mechanism of paving machines, China Journal of Construction Machinery, 7, 26-30, (2009). http://en.cnki.com.cn/Article_en/CJFDTotalGCHE200901008.htm

8 Zheng, S. H., Dai, Q. H. and Lin, S. W. Response characteristics analysis of vibrating drum in vibratory roller considering the asymmetrical hysteresis of materials, Journal of Machine Design, 33, 8791, (2016). http://en.cnki.com.cn/Article_en/CJFDTotalJXSJ201609018.htm

$9 \mathrm{Xu}$, Q., Chang, G. K. and Gallivan, V. L. and Horan, R. D. Influences of intelligent compaction uniformity on pavement performances of hot mix asphalt, Construction and Building Materials, 30, 746-752, (2012). https://dx.doi.org/10.1016/j.conbuildmat.2011.12.082

10 Amadore, A., Bosurgi, G. and Pellegrino, O. Analysis of hot mix asphalt compaction data by means of fuzzy clustering techniques, Construction and Building Materials, 40, 430-437, (2013). https://dx.doi.org/10.1016/j.conbuildmat.2012.09.082 
${ }^{11}$ Ma, J., Dong, L. J., Zhao, G. Y. and Li, X. B. Qualitative method and case study for ground vibration of tunnels induced by fault-slip in underground mine. Rock Mechanics and Rock Engineering, 52(6), 1887-1901, (2019). https://dx.doi.org/10.1007/s00603-018-1631-x

12 Dong L. J., Zou W., Li X. B, Shu W. W. and Wang Z. W. Collaborative localization method using analytical and iterative solutions for microseismic/acoustic emission sources in the rockmass structure for underground mining, Engineering Fracture Mechanics, 210, 95-112, (2019). https://doi.org/10.1016/j.engfracmech.2018.01.032

${ }^{13}$ Hou, H. B., Wang, T., Wu, S. P., Xue, Y. J., Tan, R. Q., Chen, J. Y. and Zhou, M. Investigation on the pavement performance of asphalt mixture based on predicted dynamic modulus, Construction \& Building Materials, 106, 11-17, (2016).https://dx.doi.org/10.1016/j.conbuildmat.2015.10.178

${ }^{14}$ Mollenhauer, K. and Wistuba, M. P. Influence of asphalt compaction procedure on $3 \mathrm{~d}$ deformation properties, International Journal of Pavement Engineering, 17, 5-12, (2013). https://dx.doi.org/10.1080/10298436.2013.812213

15 Tian, J. Y., Liu, G., Xiao, Z. Y. and Xu, L. Q. Vibrating character of compacting mechanism of paver, China Journal of Construction Machinery, 2, 201-205, (2004). http://en.cnki.com.cn/article_en/cjfdtotalgche200402020.htm

${ }^{16}$ Kassem, E., Liu, W. T., Scullion, T., Masad, E. and Chowdhury, A. Development of compaction monitoring system for asphalt pavements, Construction and Building Materials, 96, 334-345, (2015). https://dx.doi.org/10.1016/j.conbuildmat.2015.07.041

17 Kim, M., Mohammad, L. N., Phaltane, P. and Elseifi, M. A. Density and SCB measured fracture resistance of temperature segregated asphalt mixtures, International Journal of Pavement Research \& Technology, 10, 112-121, (2017). https://dx.doi.org/10.1016/j.ijprt.2017.01.004

18 Ma, J., Dong, L. J., Zhao, G. Y. and Li, X. B. Ground motions induced by mining seismic events with different focal mechanisms. International Journal of Rock Mechanics and Mining Sciences, 116, 99-110, (2019). https://dx.doi.org/10.1016/j.ijrmms.2019.03.009

19 Nguyen, V. L., Zhang, J. R., Le, V. Q. and Jiao, R. Q. Vibration analysis and modeling of an off-road vibratory roller equipped with three different cab's isolation mounts, Shock and Vibration, 2018, 8527574-1-852757417, (2018). https://dx.doi.org/10.1155/2018/8527574

20 Tian, G. F., Yan, W. J. and Zhang, D. W. Integrated designing method applied in paver attachment design, Journal of Shenyang University of Technology, 27, 481-484, (2005). http://en.cnki.com.cn/Article_en/CJFDTOTALSYGY200505000.htm
21 Jia, J., Liu, H. H. and Wan, Y. P. Dynamic characteristics modelling of the tamper-asphalt mixture interaction: application to predict asphalt mat density, Int. J. Pavement Eng, 20, 530-543, (2017). https://dx.doi.org/10.1080/10298436.2017.1316642

${ }^{22}$ Sun, J., Xu, G. and Wang, X. Dynamics analysis and improvement of screed based on computer simulation, Journal of Multimedia, 8, 548-556, (2013). https://dx.doi.org/10.4304/jmm.8.5.548-556

${ }^{23}$ Luo, D., Feng, Z. X. and Wang, X. Y. Parameter optimization for compacting system of asphalt paver based on response surface method, Journal of vibration and shock, 31, 92-95, (2012). http://en.cnki.com.cn/Article_en/CJFDTotalZDCJ201215020.htm

${ }^{24}$ International Organization for Standardization. Mechanical vibration and shock-evaluation of human exposure to whole body vibration-part 2: General requirements, Tech. Rep. ISO 2631-1:1997, ISO, Geneva, Switzerland, (1997).

${ }^{25}$ Nguyen, V. L., Zhang. J. R. amd Yang. X. Z. Lowfrequency performance analysis of semi-active cab's hydraulic mounts of an off-road vibratory roller, Shock and Vibration, 2019, Article ID 8725382, 15 pages, (2019). https://dx.doi.org/10.1155/2019/8725382

${ }^{26}$ Wang, W., Song, Y., Xue, Y., Jin, H., Hou, J. and Zhao, M. An optimal vibration control strategy for a vehicle's active suspension based on improved cultural algorithm, Applied Soft Computing Journal, 28, 167-174, (2015). https://dx.doi.org/10.1016/j.asoc.2014.11.047

27 Yildirim, S. Vibration control of suspension systems using proposed neural network, Journal of Sound and Vibration, 277, 1059-1069, (2004). https://dx.doi.org/10.1016/j.jsv.2003.09.057

28 Asan G. A., Hasanul B., Diyana H., et al. Semiactive vibration control using experimental model of Magnetorheological damper with adaptive fuzzy-PID controller, Smart Structures and Systems, 20, 85-97, (2017). https://dx.doi.org/10.12989/sss.2017.20.1.085

29 Ebrahimi, N. and Gharaveisi, A. Optimal fuzzy supervisor controller for an active suspension system, International Journal of Soft Computing \& Engineering, 2, 36-39, (2012). http://www.oalib.com/paper/2762439\#.XhLQG9JKjIU

${ }^{30}$ Mamdani, E. H, and Assilian, S. An experiment in linguistic synthesis with a fuzzy logic controller, International Journal of Man-Machine Studies, 7, 1-13, (1975). https://dx.doi.org/10.1016/S0020-7373(75)80002-2 